chest $\times$ ray was compatible with respiratory syndrome. A venous umbilical catheter was placed, parenteral nutrition was initiated. Minimal enteral nutrition (MEN)was started on $3^{\text {rd }}$ day of life with mother's own milk ( $1 \mathrm{ml}$ every 6 hour). She was tolerating MEN and no increment was done in the amount of milk. Until the postnatal 6th day no abdominal distension or any intolerance of MEN was detected. On postnatal 7th day there was a bluish discoloration of the abdominal wall and abdominal distension was detected. In abdominal $\times$ ray, free air under the diaphragm was detected suggestive of perforation. She was an ELBW infant in the first week of life with bluish discoloration and distention of the abdomen without any radiographic evidence of NEC as pneumatosis intestinalis or portal venous gas on the abdominal $\times$ ray. Therefore, diagnosis of SIP rather than NEC was made. The primary peritoneal drainage (PPD) was performed at the bedside due to the unstable condition of the patient. She was started inotropic support due to development of hypotension and IV antibiotic therapy was changed. Despite all the supportive therapy, the patient died on the 8 th postnatal day.

Severe placental chorioamnionitis appears to be an antenatal risk factor for SIP. A black-bluish discoloration of the abdominal wall is often seen in SIP, and is not typical of NEC. The clinical diagnosis for SIP is based upon the clinical presentation and physical findings. Abdominal radiographs that demonstrate pneumoperitoneum without pneumatosis are supportive of the diagnosis. SIP is a separate clinical entity from necrotizing enterocolitis and it is the most severe gastrointestinal complication of preterm infants. Therefore, prompt diagnosis is important because of management considerations.

\section{P471 HIRSCHSPRUNG'S DISEASE IN NEW BORN (ABOUT 17 CASES)}

${ }^{1}$ Chiraz Regaieg*, 'Dhouha Kamoun, ${ }^{1}$ Amel Ben Hmed, ${ }^{2}$ Hayet Zitouni, 'Amira Bouraoui, ${ }^{1}$ Manel Charfi, ${ }^{1}$ Nedia Hmida, ${ }^{2}$ Riadh Mhiri, ${ }^{1}$ Afef Ben thabet, ${ }^{1}$ Abdellatif Gargouri. ${ }^{1}$ Department of neonatology, Hedi Chaker Hospital, Sfax, Tunisia; ${ }^{2}$ Department of pediatric surgery, Hedi Chaker Hospital, Sfax, Tunisia

10.1136/archdischild-2019-epa.807

Introduction Hirschsprung's disease is a congenital intestinal paralysis due to absence of ganglion cells in enteric plexuses. We aim to describe the specificities of the neonatal form.

Patients and methods It is a retrospective study of 17 cases of Hirschsprung's disease hospitalized in our unit between 2006 and 2018. Diagnosis was based on radiological and/or pathological signs.

Results A male predominance was noted. Two newborns were premature. Two newborns had a congenital heart disease. One of them had Trisomy 21. Another newborn had hypothyroidism. An emission delay of méconium (average of 46 hours) was noted in all cases. The disease was revealed by a lower digestive occlusion in 7 cases, an acute enterocolitis in 2 cases and a bowel perforation in one case. The contrast enema practiced in 15 cases, was pathognomic in 13 cases. Rectal biopsy performed in 6 cases, confirmed histological diagnosis in all cases. Surgical treatment was performed in 9 cases with a median time between symptoms and surgery of 19 days. It was a colo-anal lowering in 7 cases, a resection of the right colon with double colostomy in one case and a right transverse colostomy in four cases. Outcome was favorable in 12 cases. Four newborns died consecutively to sever congenital heart disease in one case and sever sepsis in other cases.
Conclusion Hirschsprung's disease is the most common cause of digestive occlusion in the newborn. The main complications in the neonatal form are acute enterocolitis and intestinal perforation.

\section{P472 MAKING THE DELIVERY ROOM SAFER FOR NEW BABIES}

Carmel Maria Moore*, Hilda Wall, Martina Cronin, Anna E Curley. National Maternity Hospital, Dublin 2, Ireland

\subsection{6/archdischild-2019-epa.808}

Aim The 7th edition of the Neonatal Resuscitation Programme (NRP) from the American Academy of Paediatrics (AAP) is used as the safe standard of neonatal resuscitation in the Republic of Ireland. The NRP details minimum resuscitation equipment requirements. Anecdotal reports in our tertiary maternity hospital suggested a lack of adherence to this minimum equipment list, and suggested that the delivery room environment may be an unsafe place for neonates. Delayed neonatal resuscitation could have significant clinical and medicolegal consequences. Audit demonstrated poor compliance with these standards with no delivery room completely stocked. We aimed to improve this and demonstrate lasting change by improving the proportion of fully safely stocked resuscitaires in the delivery rooms.

Methods The initial audit and all subsequent re-audits were prospective and unannounced. Intervention - a laminated wall-mounted list of required equipment - was implemented by delivery unit staff. Run charts were used to monitor weekly progress.

Results Initial audit results were concerning with no resuscitaire safely stocked with all required equipment. The best stocked resuscitaire contained $88 \%$ of required equipment, with all resuscitaires missing vital equipment - including laryngoscopes, pulse oximeters and stethoscopes. The proportion of vital equipment available increased from a baseline of $66 \%$ to $98.4 \%$ the first week and $100 \%$ in subsequent weeks. The proportion of fully safely stocked resuscitaires increased from $0 \%$ at baseline to $80 \%$ the first week and $100 \%$ in subsequent weeks.

Conclusion A laminated wall-mounted checklist significantly improves the proportion of delivery rooms that are appropriately stocked with neonatal resuscitation equipment.

\section{P473 IMPETIGO IN PEDIATRIC POPULATION: A RETROSPECTIVE 6-YEAR REVIEW}

Joana Carvalho*, Sofia Aroso, Georgeta Oliveira. Hospital Pedro Hispano, Matosinhos, Portugal

\subsection{6/archdischild-2019-epa.809}

Background Impetigo is the most common skin infection in pediatric age and has the highest incidence increase relative to other skin infections observed in children. The reported incidences in the literature are between $1.65-2.8 \%$, being evident a seasonal variation with peak of incidence during summer and autumn.The aim of this study is to characterize hospitalizations for this pathology in a pediatric population covered by a level II hospital.

Methods Observational cohort study on impetigo hospitalizations over a period of 6 years between 2012 and 2017. 
Demographic data, clinical manifestations, laboratory evaluation, antibiotic therapy, duration of hospitalization and clinical evolution were analyzed.

Results During the total of this period, there were 4163 hospitalizations in the Department of Pediatrics, of which 34 $(0.8 \%)$ for cases of impetigo. Of these cases, 19 (56\%) corresponded to bullous impetigo and 15 (44\%) to non-bullous. The proportion of impetigo cases at admission ranged from $0 \%$ in 2012 to 2013, up to $1.5 \%$ of hospital admissions in 2016. Most (94\%) were term newborns, 50\% male, with a mean age at diagnosis of 12.7 days. Neonatal onphalitis was the most commonly associated pathology in 41\%. In only $26 \%$ of cases, culture of the pus or bullous fluidwas performed. The most frequent agent was Staphylococcus aureus, identified in $63 \%$ of positive cultures. The most used antibiotic therapy $(50 \%)$ was the association of flucloxacillin with gentamicin. The evolution was favorable, with complete resolution of the clinical manifestations during hospitalization, in all cases.

Conclusions Our study shows a demographic, clinical and laboratory characterization coincident with that described in the current literature. The increase in the proportion of hospitalizations due to impetigo over the years raises the possibility that the incidence of the pathology is increasing or that there is an increase in the severity of the cases. These data should be an alert on the possible influence that the adequacy of the care provided to the newborn, bothinside or outside the hospital, may have in terms of public health.

\section{P474 EXTRA-HEPATIC BILE DUCT HAMARTOMA - AN UNUSUAL CAUSE FOR ANTERIOR ABDOMINAL WALL DEFECT}

${ }^{1}$ Abhidhamma Kaninde*, ${ }^{2}$ Sheiniz Giva, 3,4 Maragaret M Moran, ${ }^{5,6}$ Salvatore Cascio, ${ }^{7}$ Erinn S McGrath, J Maureen ${ }^{8,9,10} \mathrm{O}^{\prime}$ Sullivan. ${ }^{1}$ Dept.of Neonatology, The Rotunda Hospital, Dublin, Ireland; ' ${ }^{2}$ Dept. of Neonatology, The Rotunda Hospital , Dublin, Ireland; ${ }^{3}$ Dept. of Neonatology, The Rotunda Hospital, Dublin, Ireland; ${ }^{4}$ Dept. of Neonatology, Children's University Hospital ,Templestreet, Dublin, Ireland; ${ }^{5}$ Dept. of Paediatric Surgery, Children's University Hospital, Templestreet, Dublin , Ireland; ' ${ }^{6}$ Dept. of Paediatric Surgery, Our Lady's Children's Hospital, Crumlin, Dublin, Ireland; 'Dept.of Pathology, Our Lady's Children's Hospital, Crumlin, Dublin, Ireland; ${ }^{8}$ Dept. of Pathology, Our Lady's Children's Hospital, Crumlin, Dublin , Ireland; ' University of Dublin, Trinity College , , Dublin, Ireland; ${ }^{10}$ Principal Investigator, The National Children's Research Centre,Our Lady's Children's Hospital, Crumlin, Dublin, Ireland

\subsection{6/archdischild-2019-epa.810}

We report a case of a baby girl born at 35 weeks gestation with an unusual para-umbilical lesion associated with an anterior abdominal wall defect. Shortly after birth, she was noted to have a small polypoid lesion to the right of her umbilicus. The lesion measured around $1 \mathrm{~cm}$ in length and had a small area of ulceration at the tip. Her systemic examination was otherwise normal.

Abdominal ultrasound examination was suggestive of a defect in the anterior abdominal wall. An artery and vein could be seen passing through the defect into the lesion. A rounded echogenic structure was noted to be passing through the abdominal wall defect consistent with a loop of bowel. At laparotomy, a hamartomatous lesion was seen with an artery and vein extending back to the liver. The bowel was intact and there was no bowel within the lesion. The lesion was excised, with ligation of the blood vessels, and closure of the abdominal wall defect. Screening for other midline anomalies revealed a left-sided grade 1 intraventricular haemorrhage and a small fenestrated atrial septal defect. Genetic analysis showed a normal microarray and no evidence of Beckwith-Wiedemann syndrome.

Histopathological examination of the tissue showed extensively ulcerated skin. The outline was polypoid with granulation tissue as a base. Deep to this were bile ducts, venules, and arterioles. These features were hamartomatous suggesting an extra-hepatic bile duct hamartoma. The lesion was malformative and not neoplastic.

Discussion While it is unusual to find biliary tree hamartomas presenting in the neonatal period, as most of them are asymptomatic and insidious, the extra-hepatic location of this hamartoma is extremely rare. Redston et al. reported on autopsy findings of 2843 patients and calculated the prevalence of biliary hamartomas as $5.6 \%$ in adults and $0.9 \%$ in children. They also found a high incidence of biliary hamartomas with autosomal dominant polycystic kidney disease. They can be solitary or multiple. Multiple bile duct hamartomas are also known as Von Meyenburg complexes. They are a benign ductal plate malformation of smaller interlobular ducts. Although these lesions are often described as benign anecdotally there are reports mentioning malignant transformation.

Conclusion We report a very rare case of extra-hepatic bile duct hamartoma presenting as an anterior abdominal wall defect in the neonatal period.

\section{P475 REVIEW OF THE MANAGEMENT OF BILIOUS VOMITING IN TERM NEONATES AND IDENTIFY DIFFERENCES IN MANAGEMENT OUTSIDE WORKING HOURS IN CUMH}

${ }^{1}$ Carol Stephens*, ${ }^{2}$ liam o'connell. ${ }^{1}$ Neonatology Department, cork, Ireland; ${ }^{2}$ Neonatology Department, Cork, Ireland

\subsection{6/archdischild-2019-epa.811}

Bilious vomiting is a common reason for admission to the neonatal unit. In the majority, the episode is benign. In a small minority, there is serious underlying pathology which is time critical. Identification and differentiation between these entities is crucial but challenging. Management is variable amongst centres and often case specific and certainly always time critical. When a pathological cause is suspected, occasionally it is warranted to transfer the sick neonate to a surgical unit without the delay of local investigations.

Methods This was a retrospective review in CUMH over seven years.

Inclusion criteria were all infants admitted directly to the unit with bilious vomiting.

Exclusion criteria included infants who were admitted for a different primary concern including premature infants.

Admission Logbook of unit identified infants, online reporting systems were used to review discharge summaries. Online laboratory and radiology database were accessed.

Results 213 infants were included. Management varied throughout the years. Earlier, most infants had a PFA, Septic Work Up (SWU) and contrast study in contrast to the latter years where most had a PFA and a SWU. We postulate in the latter years there was a lower threshold for admission on the background of a neonatal death and subsequently more infants were conservatively managed and observed in the unit.

$22 \%$ had contrast studies out of hours, $18 \%$ were subsequently transferred to a surgical unit in light of a positive 